\title{
Florida in a Tank- The Role of Experiments in Ecosystem Restoration
}

\section{Introduction}

South Florida is undertaking a massive restoration effort under the Comprehensive Everglades Restoration Plan (CERP), which was approved by Congress as part of the Water Resources Development Act of 2000. The primary goal of the CERP is to restore more natural freshwater flow through the South Florida ecosystem. In the last century, changes in delivery of freshwater to the estuaries of South Florida, including Biscayne Bay and Florida Bay, have altered their natural patterns of salinity. Many detrimental environmental effects, such as seagrass die-offs and loss of biodiversity, have been blamed on changing salinity. The goal of the U.S. Geological Survey (USGS) Ecosystem History Projects has been to identify natural cycles of change prior to human alteration of the environment and to distinguish natural change from anthropogenic change.

Understanding natural cycles of change and the way a system originally functioned is essential to establishing success criteria for restoration. Knowledge about how human activities and natural fluctuations affect ecosystem change can be obtained by observing potential cause-and-effect relationships in the field, forming hypotheses, and then testing these hypotheses under controlled conditions. If laboratory conditions faithfully replicate field conditions, then laboratory experiments can generate new hypotheses that can be tested by field observations.

The USGS has replicated several South Florida ecosystems in the USGS Leetown Science Center in West Virginia (figs. 1 and 2). Through laboratory studies, we hope to do the following:

- Develop new techniques for deriving water-chemistry information from organisms that secrete calcium carbonate

- Apply these techniques to document seasonal salinity changes during a century or more

- Understand the effects of salinity and other ecosystem fluctuations on submerged aquatic vegetation (including seagrass and macroalgae) and associated animal life

- Develop success criteria for CERP

Figure 1. Map of the eastern United States showing the location of a study area in Whipray Basin, Fla., and of the USGS laboratory at the Leetown Science Center, Kearneysville, W. Va., where the Florida ecosystem has been replicated. Photographs show the similarity of turtle grass (the seagrass Thalassia testudinum) in both places. Base satellite image map from the National Oceanographic and Atmospheric Administration, 2003. USGS satellite image of South Florida from 1993. Photographs of turtle grass by J.B. Murray, USGS.

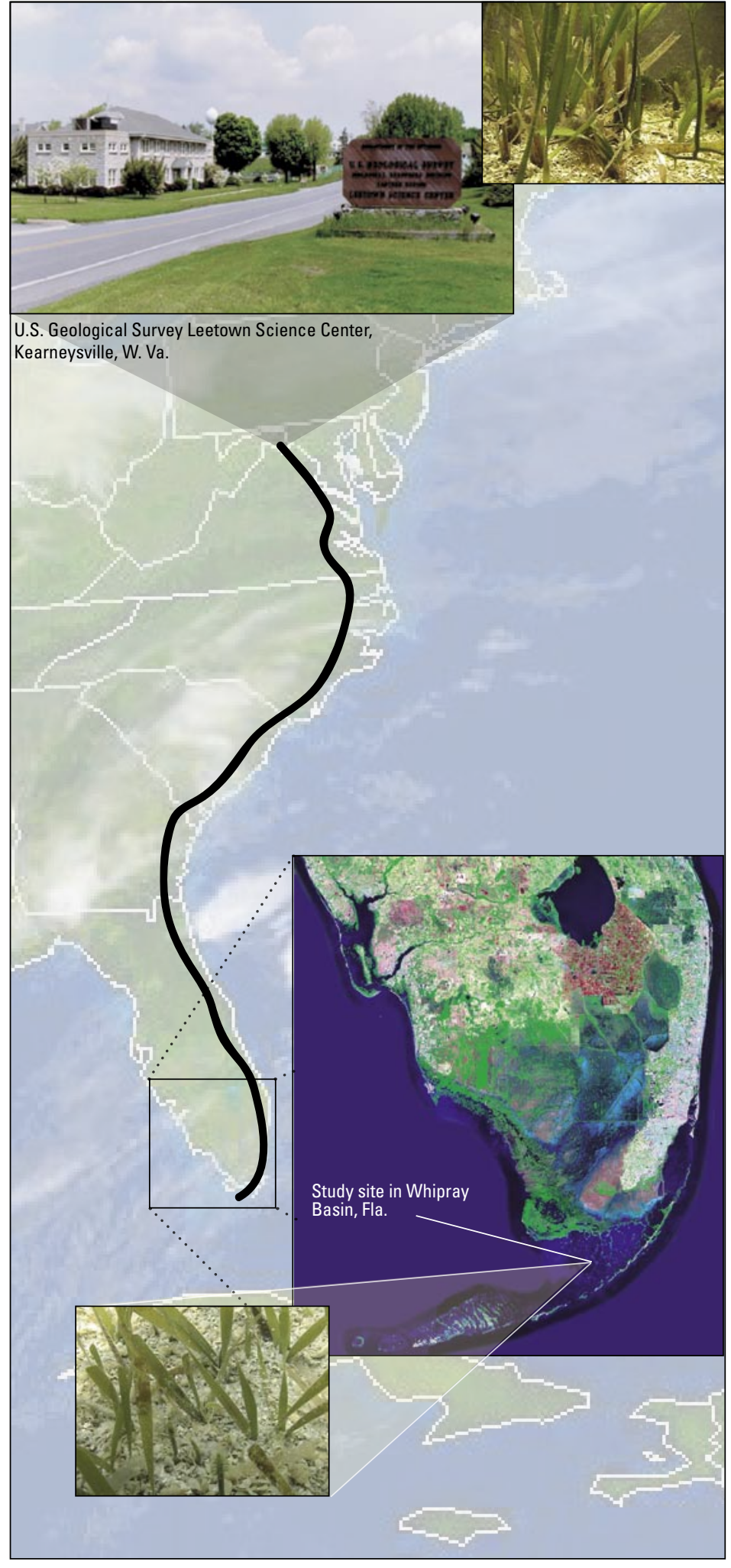




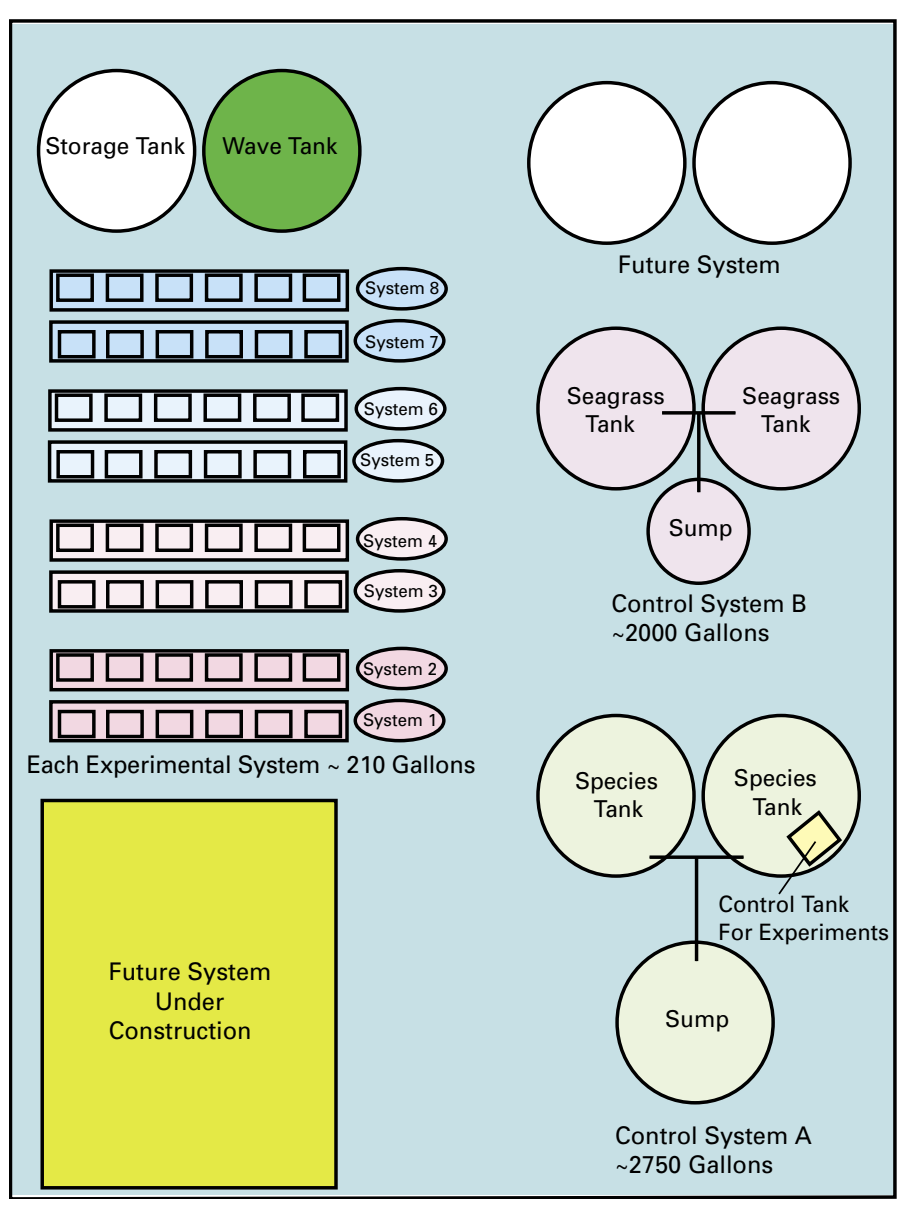

Figure 2. Schematic diagram of the tank systems in the USGS Leetown Science Center greenhouse. Control systems $A$ and $B$ reproduce the diversity found in Florida Bay. Systems 1-8 are the environments for experiments.

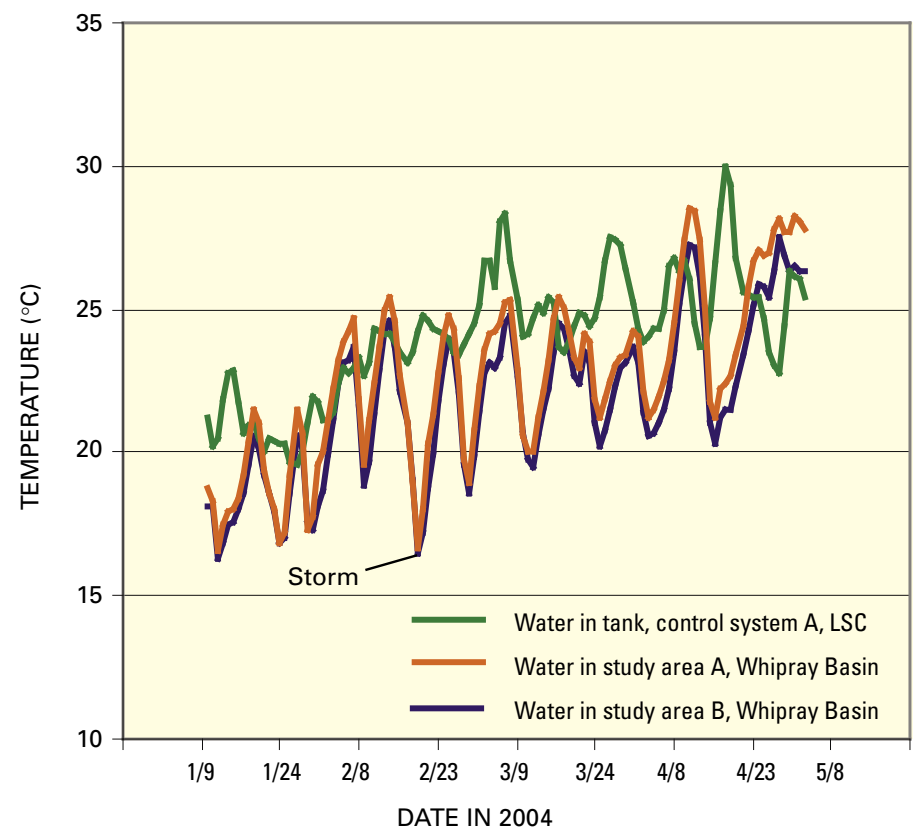

Figure 3. Comparison of water temperatures of tanks in control system $A$ in the USGS Leetown Science Center (fig. 2) with water temperatures from two data loggers ( $A$ and $B$ ) in Whipray Basin, Fla. The example data from January to May 2004 demonstrate that laboratory temperatures are very similar to those in the study area, although they do not match the low temperatures caused by storms.

\section{USGS Laboratory Systems}

Culture and test systems in tanks (mesocosms) at the USGS Leetown Science Center (LSC) were designed to replicate marine and estuarine seagrass ecosystems typical of South Florida (fig. 2). The systems at the LSC are monitored by using multiparameter water-chemistry sensors and data loggers (see box below). To provide comparison data from the real world (fig. 3), these parameters are measured at various sites in Florida Bay by water-monitoring stations installed by Everglades National Park and the USGS. Data are stored at the loggers and are downloaded to computers during periodic field visits.

The success of the LSC laboratory systems is indicated by the diversity of species that they support (figs. 4 (this page) and 5 (page 4)). Currently, the LSC systems maintain over 200 species of plants and animals, including macroalgae, submerged aquatic angiosperms (true seagrasses), many types of calcareous algae, mollusks (snails and clams), echinoderms (seastars, urchins, brittle stars), numerous cnidarians (corals, anemones), crustaceans, and an array of worms of various phyla. Examples of experiments conducted in the LSC mesocosms are provided in the boxes on page 3 .

\begin{tabular}{|llll|}
\hline \multicolumn{3}{|c|}{ Water-Chemistry Parameters } & Measured in the Tanks \\
alkalinity & dissolved oxygen & magnesium & $\mathrm{pH}$ \\
ammonia & iodine & manganese & salinity \\
calcium & iron & nitrate & strontium \\
carbon & light intensity & nitrite & temperature \\
& & & \\
\hline
\end{tabular}

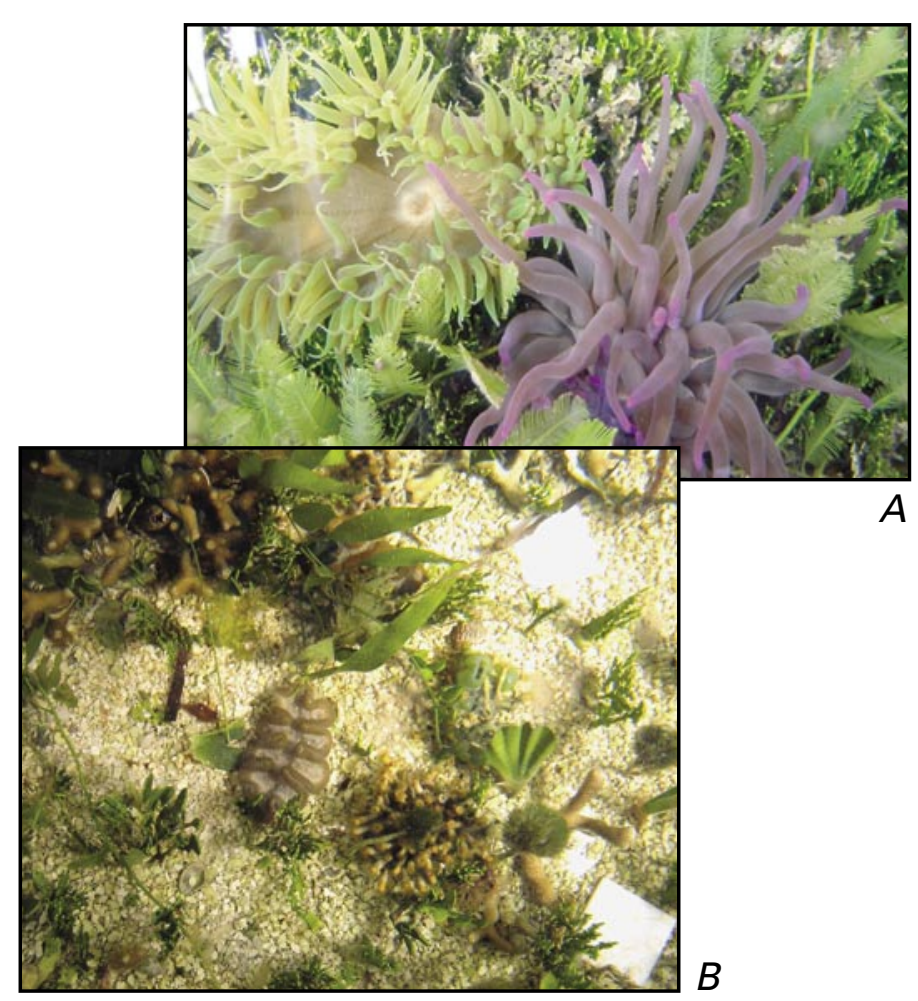

Figure 4. Images illustrating the diversity of organisms in tanks in control system $\mathrm{A}$ in the USGS Leetown Science Center (fig. 2). A, Two species of anemones and several types of macroalgae. $B$, Several species of coral and macroalgae. Photographs by W.B. Schill $(A)$ and J.B. Murray $(B)$, both of the USGS. 


\section{Experiments on Organisms that Secrete Calcium Carbonate}

Many organisms secrete calcium carbonate $\left(\mathrm{CaCO}_{3}\right)$ as they grow and thereby incorporate information about the water in which they live. The proportions of elements in seawater (for example, calcium, magnesium, barium, and strontium) are influenced by salinity and temperature and are reflected in the composition of calcium carbonate layers secreted by organisms as they grow (see figure below). We can determine historical patterns of seasonal temperature, salinity, and water-chemistry variability by analyzing the elemental composition of calcareous shells, including those of mollusks, ostracodes, and foraminifera, extracted from sedimentary layers in shallow cores.

In order to interpret these data, we must understand the biology of the living organism. What is its growth rate? What is its life span? How do environmental variables such as salinity and temperature alter the growth rate? Is growth constant? Laboratory experiments using the LSC systems test the effects of various environmental scenarios on the survival, growth, and reproduction of the shell-forming animals mentioned above as well as algae that produce calcareous skeletons. Examples of ongoing experiments include the following:

- Investigating the effects of increasing salinity and temperature; the experiment reproduces conditions caused by evaporation of warm bay waters

- Investigating the effects of a rapid influx of freshwater; the experiment reproduces conditions associated with a storm or the release of impounded waters

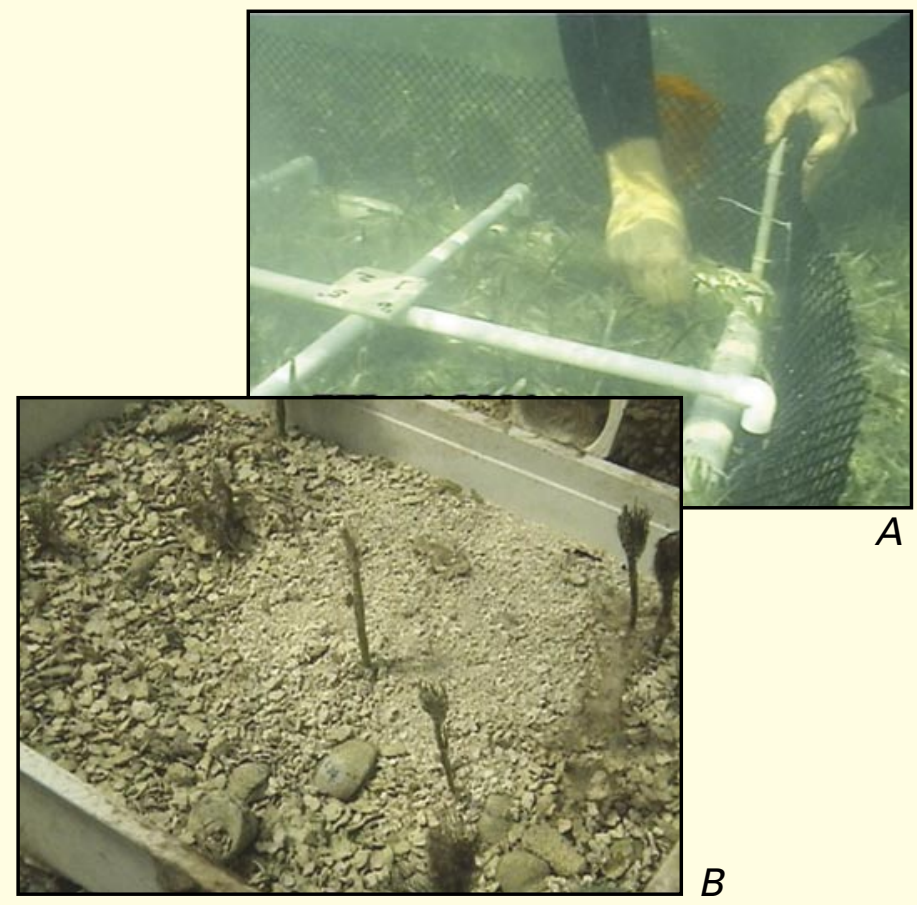

Clams (Chione cancellata) being placed in a study area in Whipray basin, Fla. $(A)$, are the same species being grown in the USGS Leetown Science Center $(B)$. The water chemistry at both sites is being monitored, and its effects on the elemental composition of the calcium carbonate shells that the clams are secreting will be determined; this information will calibrate the standards used in interpreting paleoecology from buried shells recovered in cores. Photographs by J.B. Murray, USGS.

\section{Experiments on Seagrass}

The die-off of seagrass beds is a worldwide problem and a critical issue in any estuarine ecosystem because seagrasses are essential components of those systems. Seagrass meadows provide feeding grounds, nurseries, and habitats for many forms of marine life, including such economically valuable species as shrimp, lobster, and commercially harvested fish. They also are important foraging sites for many species of migratory birds. In addition, seagrass beds anchor sediments and impede sediment resuspension and coastal erosion during storms and thus play a role in protecting other marine ecosystems, including coral reefs. The immense surface areas of the seagrass plants and the sediments underlying them (see figure below) harbor microbes that perform many important functions, including nitrogen cycling, sulfur and metal reduction, and metabolism of organic pollutants.

Seagrass beds are considered critical habitats within the South Florida ecosystem, and many hypotheses have been proposed to explain die-offs such as those that occurred in Florida Bay in 1987-88, when about 18 percent of the beds were affected. Unfortunately, seagrass biologists have lacked experimental systems to test these hypotheses under controlled conditions. As a result, the causes of these die-offs and the factors that control the health and distribution of seagrasses remain poorly understood. An understanding of these factors is essential if resource managers are to prevent further losses and restore damaged systems. We are using the culture and test systems at LSC and advanced molecular methods to study seagrass and to do the following:

- Identify disease agents associated with seagrass die-offs

- Define the role of environmental stressors (such as salinity fluctuations) in seagrass die-offs

- Investigate the role of seagrasses in estuarine biogeochemical processes

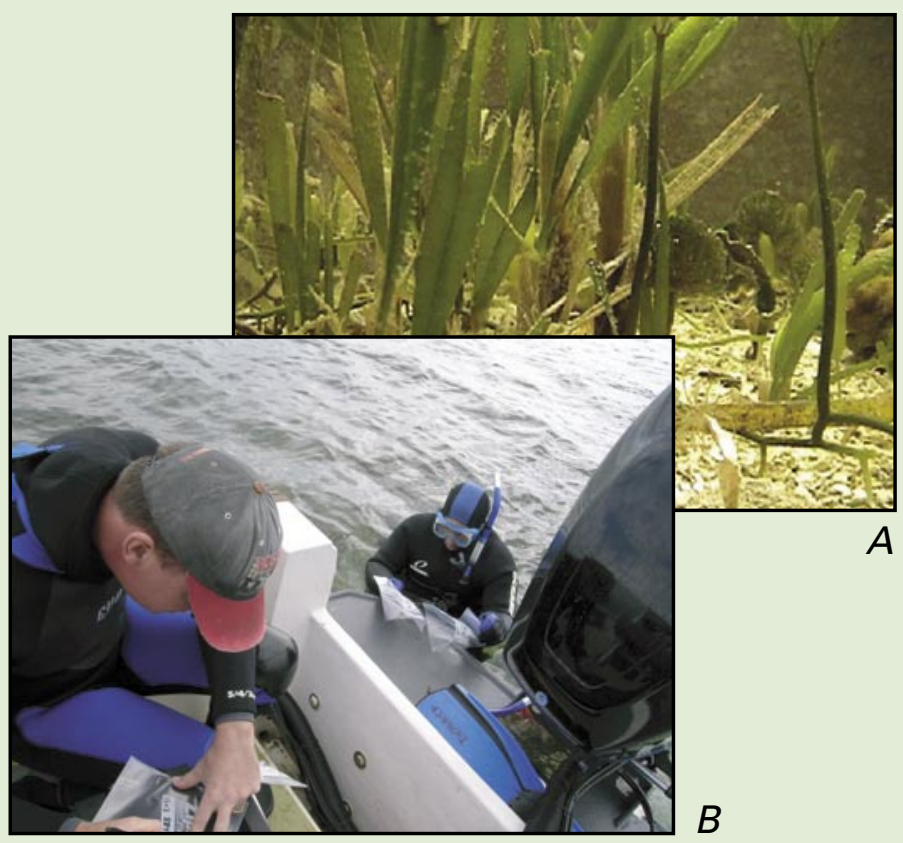

Seagrass in control system $A$ in the Leetown Science Center $(A)$ and USGS scientists collecting samples of the sediment underlying seagrass beds in Whipray Basin, Fla. $(B)$. Both the seagrass and the sediment harbor microbes that perform many important functions. Photographs by J.B. Murray $(A)$ and W.B. Schill $(B)$, both of the USGS. 


\section{Importance of Experimental Studies in Restoration}

Adaptation and selection of plants and other organisms occur over biologically significant periods of time in response to natural changes to the ecosystem. Anthropogenic changes to the South Florida ecosystem during the 20th century altered the natural patterns of change. As restoration proceeds, the natural rates of change must be considered. If restoration induces rapid rates of change to the ecosystem that exceed natural rates of change, then plants and other organisms may not be able to adapt.

Results of the experimental studies conducted in the mesocosms and results of the advanced molecular analysis, linked to the ecosystem history studies, provide insights into the rate of restoration that is tolerable by the system. These results will enable resource managers responsible for implementing the CERP to develop a model to predict future response to restoration changes. The resource managers can then set realistic targets and performance measures for restoration. Knowledge gained from this study should be applicable to other estuarine ecosystems throughout Florida and the Gulf of Mexico and, with modifications, to similar threatened ecosystems around the world.

\section{What's Next?}

Current plans to utilize the USGS Leetown Science Center mesocosms include the following studies:

- Investigations of reef-decline issues, such as coral diseases, heavy-metal contamination, pesticide contamination, and coral genetics

- Identification of the effects of invasive species within the marine and estuarine ecosystems of South Florida

- Extension of chemical analysis techniques to study the carbonate structures of marine macroalgae

By James B. Murray, William Bane Schill, and G. Lynn Wingard

For more information on the mesocosms and ongoing studies, please contact:

James Murray, jbmurray@usgs.gov

For more information on microbial and molecular studies, please contact:

Bane Schill, bane_schill@usgs.gov

\section{For more information on ecosystem history research in} South Florida, please contact:

Lynn Wingard, lwingard@usgs.gov

http://sofia.usgs.gov/flaecohist

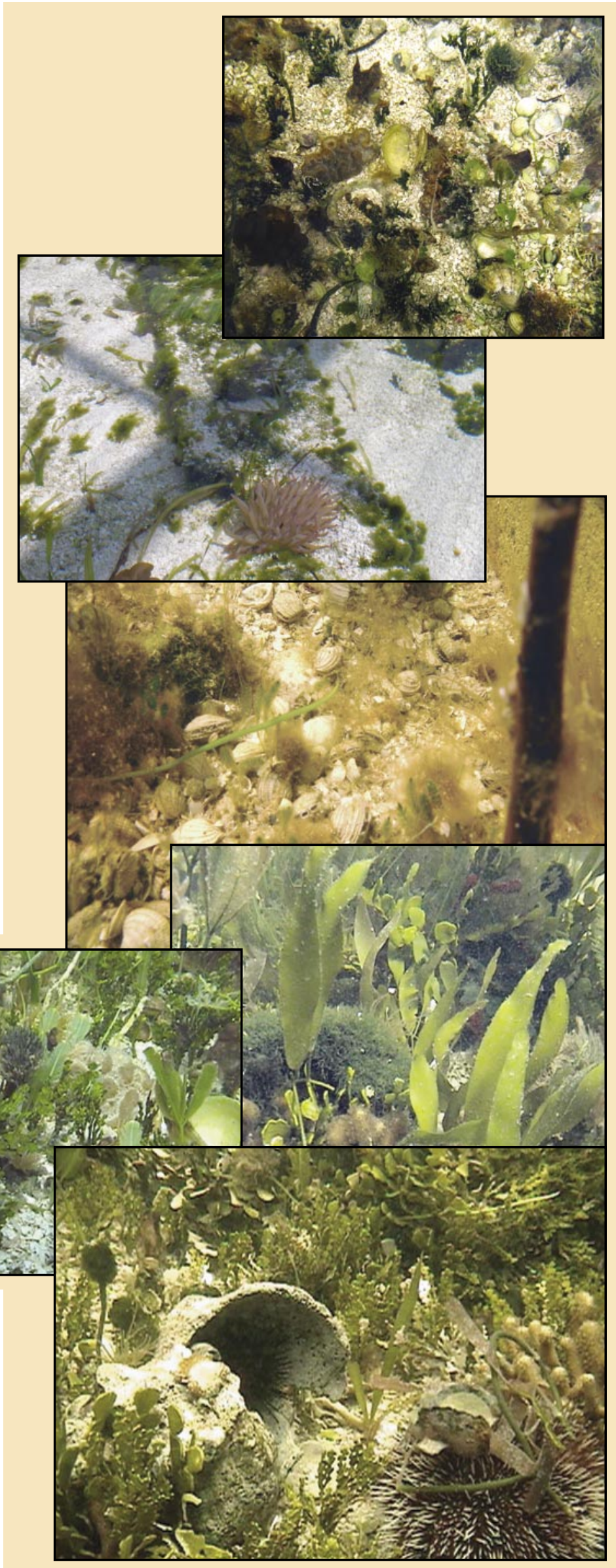

Figure 5. Images of the diverse plants and animals in the USGS Leetown Science Center mesocosms. The second photograph from the top is by W.B. Schill, USGS; all other photographs in this figure are by J.B. Murray, USGS. 